\title{
The affordance of anchored discussion for the collaborative processing of academic texts
}

\author{
J. van der Pol • W. Admiraal • P.R.J. Simons
}

Received: 1 August 2005 / Revised: 30 June 2006 /

Accepted: 30 June 2006 / Published online: 19 August 2006

(C) International Society of the Learning Sciences, Inc.; Springer Science + Business Media, LLC 2006

\begin{abstract}
A system for "anchored discussion" is compared with a system for traditional forum discussion (Blackboard), and their collaborative and communicative affordances for the collaborative processing of academic texts are investigated. Results show that discussion in the system for anchored discussion is more directed at processing the meaning of texts than discussion in the traditional forum, which is more oriented towards the sharing of personal opinions and experiences. This difference in orientation produces a more constructive collaboration in the system for anchored discussion, versus a more debate-like collaboration in the forum discussion. Additionally, while messages in the traditional forum resemble usual discussion or email conversation and contain social and regulative comments, discussion in the system for anchored discussion is seen to be more efficient and "to-the-point." We conclude that for collaborative text comprehension by undergraduate students, anchored discussion might be more suitable than traditional forum discussion. Finally, the observed differences can be explained by the stronger defined collaborative context in the system for anchored discussion, which focuses participants' collaborative intentions and their frames of reference.
\end{abstract}

Keywords CSCL - Anchored discussion - Annotation - Collaborative literature processing $\cdot$ Theory oriented discussion $\cdot$ Mutual understanding

J. van der Pol $(\bowtie) \cdot$ W. Admiraal • P.R.J. Simons

IVLOS, University of Utrecht, The Netherlands, Heidelberglaan 8,

3508 Utrecht, The Netherlands

e-mail: j.vanderpol@ivlos.uu.nl

W. Admiraal

e-mail: w.admiraal@ivlos.uu.nl

P.R.J. Simons

e-mail: r.simons@ivlos.uu.nl 


\section{Introduction}

In higher education, processing academic literature can be seen as a central but often quite challenging task, especially for undergraduate students. Assuming that a deep processing of the subject matter requires an active construction of knowledge by the learner (Boekaerts \& Simons, 1995; Phillips, 1995) for which social interaction can be helpful (Simons, Van der Linden \& Duffy, 2000), this study concerns the facilitation of collaborative literature processing. Focusing on students' collaboration as a means to learning, this study can be labeled as being based on an "interactional constructivist epistemology" (Suthers, 2005). In attempting to create a successful collaboration that involves students' active interaction with content, this study will make use of online asynchronous discussion. As Warschauer (1997) states, this medium offers the important possibility to link dialogue and interaction with individual study and reflection. Combining the advantages of social interaction, such as increased 'ownership' of ideas and the opportunity to connect to existing knowledge, with the possibilities of delayed communication for (re)reading, (re)writing, and reflection (Moon, 1999), online discussion should provide room for a thorough processing of students' course materials.

Clark, Weinberger, Jucks, Spitulnik and Wallace (2003) have remarked, however, that generic tools for online discussion still have some limitations when used in educational practice and that not all if its potential is yet being realized. Many studies report a lack of collaborative knowledge construction in online discussion. Activities that are scarcely found are integrating (Wan \& Johnson, 1994), transforming (Veerman, 2000), or discussing (Hewitt \& Teplovs, 1999) one another's ideas. More generally, students are found not to display many "higher cognitive skills" (Sringam \& Geer, 2000), or to engage much in "constructive communication" (Lipponen, 2001). Instead, Pena-Shaff and Nicholls (2004), Guzdial and Turns (2000), and Fay, Garrod and Carletta (2000) found students' communication to consist largely of independent monologues, a finding that seems consistent with that of De Laat (2002) and McLoughlin and Luca (2000), who report communication to be mainly directed at what Gunawardena, Lowe and Anderson (1997) describe as "lower-level learning activities," such as the sharing and comparing of knowledge.

\section{Supporting collaborative knowledge construction}

From the previous section we can conclude that collaborative knowledge construction is a delicate process that in many educational situations will need to be supported in order to be successful. However, deciding how or where to support it requires a thorough understanding of the process itself. Important in this respect is the study of Järvelä and Häkkinen (2000) who established a link between the depth of discussion and the subject of messages.

In their definition of different levels of discussion, they associate theory-based messages with deeper levels of constructive and cross-referenced discussion than opinion-based messages, which are associated with lower-level discussion that includes less constructive and more independent messages. Research by Van der Pol (2002) into the online collaborative processing of academic literature reports this relationship as well. In this study, it seemed especially difficult for students to 
provide each other with specific and relevant feedback to advance their understanding of the subject matter. Instead, students were more inclined to share existing experiences and perspectives, resulting in a more associative connection between consecutive messages.

This apparent preference of students for sharing opinions and experiences instead of building new understanding of the subject matter can be understood in the following way. To begin with, the mediated nature of abstract knowledge can cause learning in a university setting to differ from more direct learning as it might take place in other "real" settings (Laurillard, 1993). Since the somewhat 'unnatural' task of processing academic learning materials might not lie very close to the personal perspectives of the participating students, a link might need to be negotiated between students' personal and more academic perspectives (Petraglia, 1998). Furthermore, constructing new knowledge is a difficult process, as students need to 'discover' a new understanding of the subject matter that no one yet possesses individually. As Stahl (2000) illustrates in his model of "social knowledge building," students' personal understanding or better, their "tacit pre-understanding" forms an essential input in the social knowledge-building cycle. A possible effect of a limited personal understanding of the subject matter is that both articulating one's questions and interpreting those of others and providing them with specific and relevant feedback will require a high amount of mental effort. While this meaningprocessing effort seems to be exactly what drives learning (Baker, Hansen, Joiner \& Traum, 1999; Schwartz \& Lin, 2000), it also might sometimes exceed students' capabilities. Instead, sharing existing opinions and experiences seems to be a lessdemanding option for students to participate in online discussions.

Especially in electronic environments, it seems important to monitor the amount of effort that is required for successful collaboration as the medium is limited in supporting the development of mutual understanding, or "grounding" (as defined by Clark \& Brennan, 1991). Not only does inferring the perspective of the future reader during message formulation ("audience design") seem to be more difficult in manyto-many communication than in two-party conversations (Gergle, Kraut \& Fussel, 2004), but, as argued by Fussel and Benimoff (1995), several additional features of online discussion, such as its delayed feedback, low amount of "turn-taking" and lack of non-verbal clues, make it hard to repair miscommunication and maintain a "shared communicative context." Although grounding can be seen as a functional process (Clark \& Brennan, 1991; Dillenbourg, 1999), meaning that the amount of grounding activity generally will match the need for it, Gergle et al. (2004) demonstrated that with regard to the communicative efficiency of computer-mediated communication (CMC), these compensations often fall short.

Although the complex processes of successful collaborative learning may require more than just a strong link between discussion and subject matter and efficient communication, they can be seen as important prerequisites for a successful collaborative processing of literature, and we will now direct our attention towards finding ways to facilitate these two processes. One way to focus the collaboration and grounding efforts of students would be to change the pedagogical approach. However, introducing additional training or elaborate instructions could increase the already high demand of time and effort in online discussion for both students and teachers. We believe, in line with Dillenbourg (1999), that grounding efforts should remain subordinated to the accomplishment of the task and the production 
of "rich" interactions; it would then seem important to make sure students' efforts are invested in the most optimal and productive way. To accomplish this, our study investigates how overcoming the two identified obstacles could be afforded by features of the electronic environment itself. We feel this could be a fruitful approach as the functionality of the generic discussion tools generally being used in university settings have not specifically been developed to support a collaborative processing of literature.

We will specifically investigate whether a tool's functionality can influence students' collaboration implicitly, thus preserving an 'open' learning environment that does not restrict users' actions.

\section{A specialized design: Anchored discussion}

Hunt (1998) maintained that the fact that students in older bulletin board systems appeared to respond immediately and without much reflection was the effect of a lack of context. In line with this thought, we will investigate a tool for "anchored discussion" (see Bernheim Brush, Bargeron, Grudin, Borning \& Gupta, 2002), trying to overcome some of the limitations of asynchronous electronic communication as mentioned in the introduction. Anchored discussion (Figure 1) finds its roots in literature processing-oriented systems for shared annotation (see Davis \& Huttenlocher, 1995; Sumner \& Buckingham Shum, 2001), yet takes a slightly different approach. While shared annotation starts from the notion of personal annotation

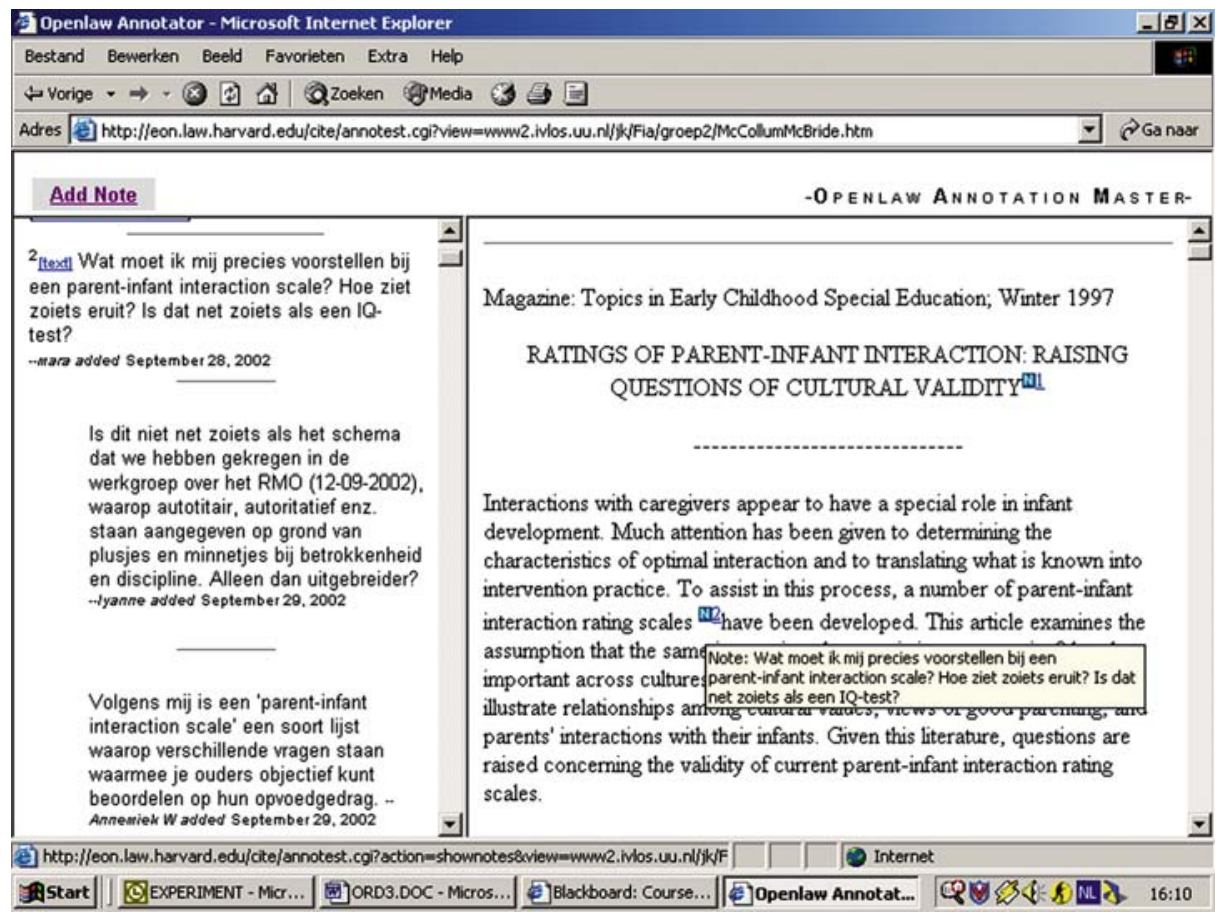

Figure 1 Adapted version of the "Openlaw Annotation Master" (Seltzer, 2000), presenting both the "threaded" discussion (left) and the article under discussion (right) 
made visible to peers, anchored discussion starts from the notion of collaborative discussion that is contextualized or "anchored" within a specific content. While systems for shared annotation are inclined to display individual notes within the text (as in Schoonenboom, 2002) anchored discussion, or "linked artifact-centered discourse," displays both artifact and discussion in a linked, yet independent manner (Takeda \& Suthers, 2002). An effect of this difference in origin and interface is that shared annotation might leave more room for individual processes, but is shown to have some limitations in supporting interactivity. Nokelainen, Miettinen, Kurhila, Floréen, and Tirri (2005) found a positive relation between an individual learner's activity in a system for shared annotation and their study success, but they also established a possible distracting effect of shared annotation as users viewed self-made highlights and comments as being more useful than those made by other learners. Comparably, a large-scale study by Cadiz, Gupta, and Grudin (2000) on a system for shared annotation showed that the majority of annotations did not contain any replies, whereas studies by Bernheim Brush et al. (2002) and Guzdial and Turns (2000) showed that threads in a system for anchored discussion were significantly longer than those in regular forum discussion. Although a tool's effect on the quantity of interaction highly depends on its pedagogical implementation and particular functionality (see Marshall \& Bernheim Brush, 2002), these results indicate that the interaction-oriented design of anchored discussion could offer good possibilities for supporting students' collaborative processing of academic texts.

While several of the studies mentioned above determined anchored discussion to be a potentially valuable medium for collaborative learning, they do not directly investigate its effects on the quality of interaction, or compare this to other tools for collaborative learning. Therefore, this study will aim to compare a system for anchored discussion with a system for traditional forum discussion and to investigate their collaborative and communicative affordances.

\section{Anchored discussion versus forum discussion}

Having presented anchored discussion as a viable alternative for regular online discussion, we will now describe how we expect their respective functional differences to influence the collaborative learning processes and to possibly support students' collaborative processing of scientific texts. Looking at the functional differences between a system for regular forum discussion (Figure 2) and the system for anchored discussion used in this study, we see that the system for anchored discussion integrates a document or text into the online discussion environment in two ways. Apart from physically presenting the text online, adjacent to the threaded discussion, it offers the possibility to anchor messages to specific passages of the text.

The visual integration of text and discussion in the system for anchored discussion might present the most obvious difference from "regular" forum discussion. As to the effect of this integration, Herrmann and Kienle (2003) mention that material that is provided in computer-based collaborative learning environments can and should serve as "context for collaboration" In line with this thought, Gay, Cornell, Sturgill, Martin and Huttenlocher (1999) state that document-mediated communication can define a stronger collaborative context, setting the orientation and providing a means for effective communication. In other words, the explicit 


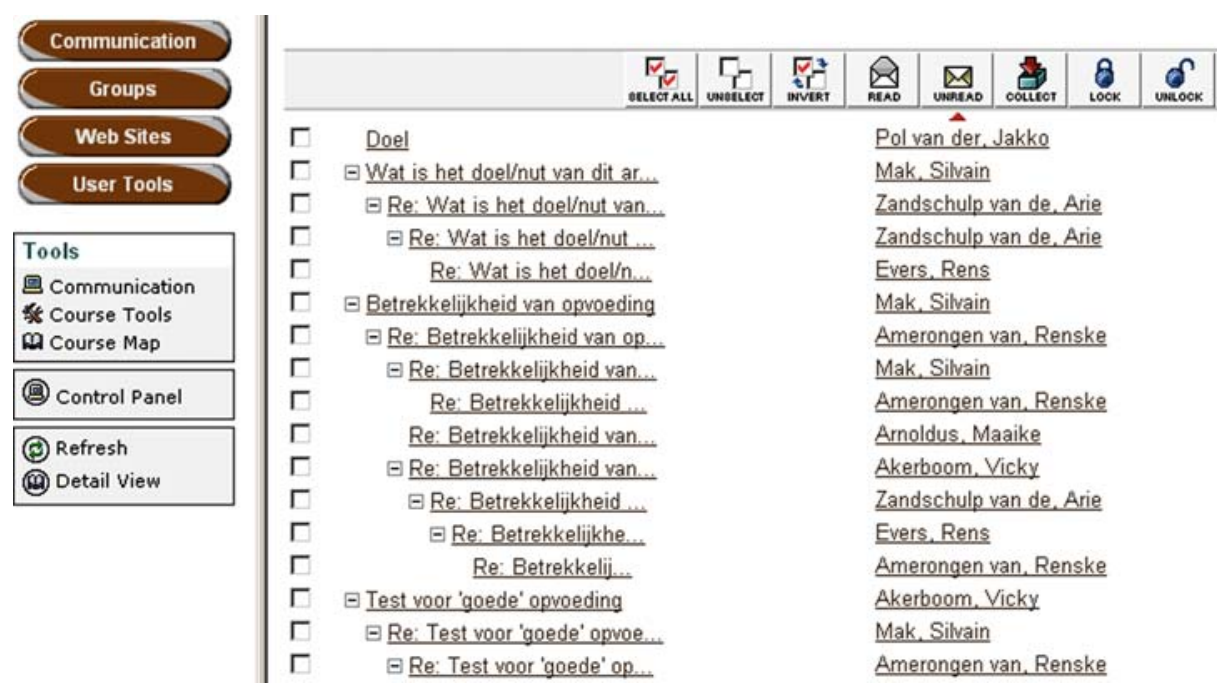

Figure 2 System for regular forum discussion (Blackboard) that was used in this study

document-centeredness of anchored discussion might naturally direct users' collaborative intentions towards the processing of that text. According to Guzdial and Turns (2000), this could strengthen the link between discussion and study material and make the discussions more effective. Additionally, this automatic focus of students' intentions and perceptions of the collaborative goal might also reduce the need for coordination of the collaborative process.

The possibility of anchoring messages to specific passages of the text can provide individual messages or threads with a stronger "frame of reference." As Herrmann and Kienle (2003) describe, being able to refer to a piece of available context will reduce the level of explicitness that is required. Additionally, as described in Clark and Brennan's (1991) "principle of minimal effort," this available frame of reference can be expected to reduce the "space for misunderstanding" (Dillenbourg, 1999), facilitating interpretation and requiring students to invest less effort in clarifying their messages.

\section{Research questions}

To investigate whether the design of the system for anchored discussion supports the collaborative processing of academic texts more than a system for traditional forum discussion this study focuses on four concrete research questions. The first two aim to assess whether the system for anchored discussion strengthens the link between discussion and text and enhances the efficiency of communication. The last two questions aim to check the general suitability of the system for anchored discussion for supporting online discussion.

Do the two systems:

1. Differ in their ability to strengthen the link between discussion and study material? 
2. Support different levels of communicative efficiency?

3. Produce a different kind of conversation?

4. Provide different constructive activities?

\section{Materials and methods}

\section{Research context}

This study was conducted in a Dutch first-year first trimester pedagogy course titled "General Pedagogy". In this course, the students collectively received weekly lectures and participated in weekly seminars in subgroups. In total, the 193 students enrolled in the course were divided into nine seminar groups with six teachers. During the course, the students had to read several English articles and discus them in the seminars. The reading for the course was planned week by week. To stimulate students' processing of the course material, the course provided an online facility for collaboration. This voluntary online collaboration took place either in a "regular" Blackboard discussion forum (see Figure 2), or in the system for anchored discussion described above (see Figure 1). To control the influence of the seminar teacher in the two conditions, each seminar group was randomly split into two subgroups, each of which was assigned to one of the conditions.

Two articles were covered in a two-week online discussion round (starting in week 1 and 3), prior to their face-to-face discussion in the seminars. The students were instructed to try to help each other develop a better understanding of the text by asking each other questions about difficult passages and lines of reasoning and by trying to explain to each other how these can be understood. As a reward for sufficient participation (submitting at least 2 messages per week), students were allowed to skip a question on the final exam. The discussions were moderated by the seminar teachers (most of whom were inexperienced ICT users), who received instruction by the researcher. This instruction not only served to demonstrate the electronic environments, but also to optimize the teachers' pedagogical implementation of them in the course and provide an optimal base for collaboration in both conditions. The educational advantages of asynchronous electronic discussion were discussed, and several strategies to increase the chances of creating successful discussions, as described in Pena-Shaff and Nicholls (2004) and Van der Pol and Admiraal (2003), were highlighted. The instruction emphasized the medium's potential to make students' intuitive understanding explicit, which enables the teachers to connect to them. It was also stated that to encourage students to express their intuitive and uncertain ideas and questions about the text, a constructive and helpful conversation may be more effective than a sharp and critical debate. Concerning moderation of the discussions, we suggested the teachers not be too authoritative, but rather to respond to students' difficulties with follow-up questions and to try to support their advancement in small steps.

\footnotetext{
${ }^{1}$ The term "pedagogy," in Dutch, is used to refer to the science that is concerned mainly with educating or rearing children, and deals with normative questions such as what to learn (opposed to the more instrumental approach of educational science that is directed mainly at how to learn).
} 
The electronic messages of the discussions, or "collaboration protocols," will serve as the main research data, complemented information from questionnaires and group interviews. With nine seminar groups, each containing two online discussion groups (one for each condition), 18 student groups were simultaneously discussing the same course materials for a 2-week period. This was done twice, resulting in a total of 36 discussions with a total of 1,133 non-accidental, student-generated, taskrelated messages. After finishing the discussions, students were asked to complete a questionnaire and four group interviews were conducted. In total, 111 questionnaires were fully completed, 62 for the anchored discussion condition and 49 for the regular forum discussion condition. Two discussion groups from each condition were interviewed, with approximately ten students each.

These three data sources will be used to address the research questions in the following way. First, the subjects of messages and the number of references to the subject matter (collaboration protocols) and students' reported off-line reading activities (questionnaire), will be used as indicators for the link between discussion and study material. Second, the communicative efficiency will be measured in terms of the elaborate or demonstrative nature of references, with the need for selfclarification within individual messages and students' reported levels of mutual understanding being evaluated. Third, the nature of conversation will be investigated using the frequency of social and regulative comments and the average number and length of messages. These protocol data are completed by students' experiences with the two tools, as collected in the group interviews. Fourth, the constructive activities are based on the number and type of questions, answers and critical reactions ("message type"), as well as the number of argumentations, confirmations and clarifications. Finally, additional data from the protocols, interviews and questionnaires will be used to check whether the two systems were easily used and if they presented any major technical difficulties that might have interfered with the investigation of their hypothesized affordances.

\section{Measures}

\section{Collaboration protocols}

In order to analyze the content of the collaboration protocols, an instrument has been developed. While many instruments already exist in this domain, they were not found to optimally suit our specific research context and questions. The main reasons for this consideration were that theory-based models did not seem to fit our practical reality of students' collaborative knowledge building when possessing only low levels of expertise. As these students do not generally follow a scientific cycle of inquiry, it is important not to overlook "where students are at" (Pilkington, 2004) and at how students in practice engage in constructive conversation. Other instruments, like the coding scheme of Järvelä and Häkkinen (2000) do seem to fit better our context, but were found to present difficulties when trying to establish sufficient levels of inter-rater reliability. Therefore, a new coding scheme was developed that, instead of presenting a measure for the overall quality of a discussion, was aimed at identifying several separate and more basic characteristics 
of the discussions. This development was partly done in a top-down manner, based on existing coding schemes, and partly in a bottom-up approach, grounded in the collected data. Messages were used as the unit of coding. The categories that were created cover the variables "message subject," "message type," "argumentation," "confirmation," "clarifying," "referring" and "social" and "regulative" comments (see Table 1). The inter-rater reliability, in terms of Cohen's kappa, exceeded 0.70 for all categories, with an average of 0.82 . Due to a low reliability, the connectivity (the relevance of a response in relation to the message it replies to) has not been included in further analysis.

Regarding the subjects of messages, we have identified three main categories that describe whether a message is concerned with the meaning of the article ("What does the author want to say in this article?"), students' opinion ("What is the student's personal opinion about certain issues in or related to this article?"), and whether the message is concerned with the task of processing the article at all (nontask-related). Furthermore, since anchored discussion has been found to produce discussion that is more focused on specific concepts than regular forum discussion (Bernheim Brush et al., 2002), the messages oriented at establishing the meaning of the article are further coded on how general or local is the issue they

Table 1 Instrument for dialogue analysis

\begin{tabular}{|c|c|c|c|c|c|}
\hline \multirow{3}{*}{$\begin{array}{l}\text { Variable: } \\
\begin{array}{l}\text { 1) Message } \\
\text { subject }\end{array}\end{array}$} & \multicolumn{3}{|l|}{ Categories: } & \multirow{2}{*}{$\begin{array}{l}\text { Type } \\
\circ\end{array}$} & \multirow{2}{*}{$\frac{\text { Cohen's K }}{0.79}$} \\
\hline & \multirow{5}{*}{$\begin{array}{l}\text { The article's } \\
\text { meaning }\end{array}$} & \multicolumn{2}{|l|}{ Overall } & & \\
\hline & & \multicolumn{2}{|l|}{ Structure } & $\circ$ & \\
\hline & & statement & argumentative & $\circ$ & \\
\hline & & & non argumentative & o & \\
\hline & & concept & & 0 & \\
\hline & \multicolumn{3}{|l|}{ opinion of student } & o & \\
\hline & non task-related & & & o & \\
\hline \multirow[t]{8}{*}{ 2) Message type } & \multirow[t]{4}{*}{ thread openers } & \multirow[t]{2}{*}{ statement } & definite & $\circ$ & 0.91 \\
\hline & & & open & ○ & \\
\hline & & \multirow[t]{2}{*}{ question } & full & o & \\
\hline & & & empty & 0 & \\
\hline & critical reaction & & & o & \\
\hline & \multirow[t]{2}{*}{ follow-up question } & \multirow{2}{*}{\multicolumn{2}{|c|}{$\begin{array}{l}\text { full } \\
\text { empty }\end{array}$}} & $\circ$ & \\
\hline & & & & $\circ$ & \\
\hline & \multicolumn{3}{|l|}{ answer/ reaction } & $\circ$ & \\
\hline \multicolumn{4}{|l|}{ 3) Argumentation } & $\square$ & 0.82 \\
\hline \multicolumn{4}{|l|}{ 4) Confirmation } & $\square$ & 0.83 \\
\hline \multicolumn{4}{|l|}{ 5) Clarifying } & $\square$ & 0.75 \\
\hline \multicolumn{4}{|l|}{ (Connectivity) } & $\square$ & $(0.36)$ \\
\hline \multicolumn{4}{|c|}{ 6) Referring to a person } & $\square$ & 0.91 \\
\hline \multicolumn{4}{|c|}{ 7) Referring to the content of another message } & $\square$ & 0.77 \\
\hline \multicolumn{4}{|c|}{ 8) Referring to the content of the text } & $\square$ & 0.79 \\
\hline \multicolumn{4}{|c|}{ 9) Comprehensively referring to a location in the article } & $\square$ & 0.79 \\
\hline \multicolumn{4}{|c|}{ 10) Demonstratively referring to a location in the article } & $\square$ & 0.73 \\
\hline \multicolumn{4}{|c|}{ 11) Social comments } & $\square$ & 0.90 \\
\hline \multicolumn{4}{|c|}{ 12) Regulative comments } & $\square$ & 0.84 \\
\hline
\end{tabular}

'Type' shows whether only one of multiple categories is to be scored (expressed by an option button), or whether it is simply a 'yes/no'-variable (expressed by a checkbox). 
concern: Ranging from the overall idea and structure of the article, to individual statements (with or without argumentative reasoning), to the meaning of specific words or concepts.

The category "message type" includes whether a message is identified to start a new thread ("thread-opener"), to pose a new question within an existing thread ("follow-up question"), to express a form of disagreement or doubt ("critical reaction"), or to constitute another type of reaction or answer. When a thread-opener concerns a statement, this is scored to be either expressed as being a sure thing ("definite") or in a more careful way ("open"). When it concerns a question, it is scored as containing a possible answer ("full") or not ("empty").

Having labeled the subject and type of the messages, messages are coded for the constructive activities "argumentation," "confirmation" and "clarifying," with descriptions based on the coding schemes of Veerman (2000) and VeldhuisDiermanse (2002). These three variables contain activities that are scored as being either present at least one time in a particular message (1) or not at all (0). Thus, it is possible for a message to contain both a supporting argument ("argumentation"), an expression of agreement with someone else's earlier statement ("confirmation") and an explanation of what they mean by this ("clarification"). Note that clarification is used here to indicate when students explain what they meant by something they wrote earlier in the same message (such as paraphrasing oneself, or giving an example) and not in the sense of explaining ideas from earlier in the discussion.

To provide further information about how discussion messages are linked to the article and subject matter, students' referring activities are coded as referring to other persons ("I don't think Jan is right"), as referring to the content of either messages or the text of the article ("I don't think that is true"), or as referring to a specific location in the article in either a long and comprehensive manner ("On page 2 just below the first paragraph") or a short and demonstrative manner ("Here"). The latter distinction was used because the use of demonstrative expressions can give information about what contextual information participants consider to be evident and shared with their peers.

Finally, messages have been coded on the occurrence of social and regulative comments (cf., Veldhuis-Diermanse, 2002). Social comments comprise expressions such as "Hello everyone" and "Goodbye/good luck" and regulative comments expressions like "Can anyone help me with this question?". Social comments are used to establish and support social relationships and regulative comments function to coordinate the discussion process.

\section{Questionnaires and group interviews}

The questionnaire (see Table 2) was used to gather information about the usability of the tools, the perceived quality of the discussion, students' offline reading activities, their perceived levels of (mutual) understanding and their perception of the learning gains of the discussions. These processes were not directly visible in the protocols. Each item was intended to measure different information. The group interviews aim to get a feel for how the tool and study are experienced by the students, leaving room for a variety of input. They questioned students about the usability of the tools and their implementation in the course and focused on identifying possible disturbing factors for the design and results of the study. 
Table 2 Questionnaire items (for this purpose ordered in themes)

Link between discussion and study material:

1. How much of the article did you read before starting to participate in the discussion?

2. "Before I reply to a message, I always reread the relevant passage from the article."

Communicative efficiency:

3. "It was easy to understand the questions and answers of others."

4. "Others usually understood very well what I was trying to say or ask."

5. "In the discussions, there was a lot of miscommunication."

Usability of the tool:

6. "I find the discussion tool practical for discussing the article online."

7. "I experienced technical difficulties with the discussion tool."

8. How many of the total number of discussion messages did you read?

All measured as 5-point Likert type items; for items 1, 2 and 8, $1=0-20 \% ; 2=21-40 \%$; $3=41-60 \%$; $4=61-80 \%$; and $5=81-100 \%$, while the remaining items ranged from $1=$ "strongly disagree" to $5=$ "strongly agree").

\section{Analyses}

The unit of analysis is discussion level. This means that the data has been aggregated to be able to identify the characteristics of the discussion. In this, we corrected for the length of the discussion. $T$-tests, with an $\alpha=0.05$, tested the differences between the condition for regular forum discussion (with a score of 0 ) and the anchored discussion condition (with a score 1). For the variable's message type and subject, we divided this alpha by the number of categories to compensate for the larger number of statistical tests involved. In order to explore additional insights into the relation between tool (forum or anchored discussion), subject, and students' constructive activities, regression analyses have been performed.

\section{Results}

Use of the systems

Even though most students had no experience with either of the systems and their participation was voluntary, both discussion systems were frequently used, resulting in 514 messages in the system for forum discussion and 782 in the system for anchored discussion (excluding duplicate messages that are caused by accidentally clicking the send-button). The relative number of task-related messages in the two systems does not differ significantly ( $90 \%$ for the forum and $92 \%$ for the anchored discussion). In the questionnaires, students did not report significant differences in technical difficulties with the two tools (with means of 1.34 in the forum and 1.70 in the anchored condition), or in the extent to which students found the tool practical to use (with means of 3.57 in the forum and 3.34 in the anchored condition). Furthermore, students did not report significant differences between the conditions in the percentage of messages that were read (with means of 4.43 in the forum and 4.43 in the anchored condition). Finally, the results from both the discussion protocols and the group interviews did reveal some technical issues with the system for anchored discussion (such as the placement of certain buttons) that should be 
Table 3 Percentages of meaning-oriented conversation and objects of referrals by condition

\begin{tabular}{lll}
\hline & Forum discussion & Anchored discussion \\
\hline 1) Message subject: the article's meaning & 58 & 77 \\
6) Referring to persons & 34 & 13 \\
7) Referring to content of another message & 0 & 5 \\
8) Referring to content of the text & 5 & 12 \\
\hline
\end{tabular}

Percentages in all tables with variables from the coding scheme represent the number of messages containing the indicated activity in relation to the total number of messages within that condition. Variable numbers match the numbering as found in Table 1.

resolved in any further development, but which did not seem to have hindered constructive use of the system.

Link between discussion and study material

First, the link between discussion and study material is indicated by the subject of conversation. The two systems show a significant difference in the extent to which discussions are focused on the meaning of the article (see Table 3), $t(22.46)=2.29, p=$ 0.032. Second, the link between discussion and study material becomes visible in the object of referring activities. While discussions in the forum discussion more often contain referrals to persons, $t(34)=6.10, p<0.001$, discussions in the system for anchored discussion contain more direct referrals to actual content (ideas or statements) of other messages, $t(31.14)=-3.13, p<0.01$ and of the text, $t(20.42)=$ $-3.88, p<0.01$. Third, while the questionnaires did not reveal a significant difference in the amount of literature the students had read before starting the discussion, they did show that the tool influenced students' reading activities. Students who used the system for anchored discussion $(M=3.58, \mathrm{SD}=1.02)$, reported to have reread the relevant section of the article before replying to a

$7[$ text] What is meant by the word 'dyad'. I read it later in the article too, but my dictionary doesn't know it.

.. student $A$ added October 1, 2002

my dictionary says: couple -- student B added October 1, 2002

But then I still don't get its use here. They are talking a lot about 'parents' en 'dyads', so with 'dyad' they can't mean two parents. Then what do they mean by it

-. student $A$ added October 2, 2002

I think with 'dyad' they mean the dyad parent-child. If you assume that, it makes sense in the text -. student $C$ added October 6,2002

That could be right. I must say it is farfetched, but now I do understand it better. -- student $A$ added October 9, 2002

Figure 3 Thread from the system for anchored discussion, using demonstrative expressions 
Table 4 Percentages of types of referrals and clarifications by condition

Forum discussion

Anchored

discussion

5) Clarifying

20

9) Comprehensive referring to a location in the article 15

10) Demonstrative referring to a location in the article 5

message more often than those in the system for forum discussion $(M=2.77, \mathrm{SD}=$ 1.13), $t(108)=-3.94, p<0.001$.

\section{Communicative effectiveness}

Regarding the clarification activities (see Table 4), we see that the students in the regular forum discussion use more clarifying statements, $t(34)=5.48, p<0.001$. Table 4 also shows that in the forum discussion we find more referrals that use comprehensive expressions (references that can be understood on their own without contextual information), $t(15.59)=4.52, p<0.001$, whereas in the system for anchored discussion we find more demonstrative referrals (that are more brief because of the use of expressions such as "here" or "that," as can be seen in Figure 3), $t(29.87)=-3.95, p<0.001$. Concerning the effects of these clarifications and referring efforts, the questionnaires do not present a significantly different level of mutual understanding in the two conditions.

Nature of discussion

In the forum discussion, the structure of messages generally resembles the structure of emails, with introductory and closing parts that include more than the actual question or remark about the subject matter (see Van der Pol, Admiraal \& Simons, 2006, for examples and a more elaborate description). Messages in this condition also more often contain social statements, $t(13.82)=4.46, p=<0.01$, and regulative comments, $t(14.49)=3.91, p<0.01$, as can be seen in Table 5 .

The fact that the system for anchored discussion shows less social and regulative comments also expresses itself in the average message length. Taking the complete collection of messages $(n=1,342)$, we find that messages in the forum discussion have an average length of 57 words (see Table 6), whereas their counterparts in the system for anchored discussion, on average, consist of 38 words, $t(635.51) 2=14.17$, $p<0.001$. However, a significantly larger number of messages in the system for anchored discussion $(t(28.49)=-2.13, p=0.04)$ almost completely compensates for this shorter average message length.

Finally, the group interviews revealed a slight difference between the systems with regard to the type of discussion it best supports. While the system for anchored

Table 5 Percentages of social and regulative statements by condition 
Table 6 Average message length and number of messages per discussion by condition

\begin{tabular}{lll}
\hline & Forum discussion & Anchored discussion \\
\hline Words per message & 57.1 & 37.7 \\
Messages per discussion & 25.6 & 37.3 \\
\hline
\end{tabular}

discussion was experienced as particularly useful for the discussion of specific statements and concepts, the forum discussion was found to be better suited for more general discussion. However, the protocol analysis of the message subjects revealed no differences between the systems in the degree to which discussions are dedicated to the overall meaning of a text or to the meaning of specific concepts, nor in any of the other subcategories of the variable "message subject".

\section{Constructive activities}

Scores on the subcategories of the variable "message type" (2) did not differ significantly across conditions. However, the traditional forum discussion does show relatively more argumentations $(t(34)=3.25, p<0.01)$ and confirmations $(t(34)=$ $2.19, p=0.036$ ) than does the system for anchored discussion (see Table 7).

Expecting that it might be more difficult for students to support why they think that a passage from a text has a particular meaning than to support why they have a certain personal opinion, we checked the data for a relation between message subject and argumentation and found there is indeed less argumentation in meaning oriented discussion than in opinion-oriented discussion $(r=-0.61, p<0.001, n=36)$. To examine whether the different level of argumentation in the two conditions, as established earlier, might be mediated by this relation, a linear regression analysis was conducted (as the data fit linear models) with both message subject and tool as predictors for argumentation (see Table 8). From this, we find that the correlation between condition and argumentation decreases from 0.49 to 0.30 when controlling for message subject, which confirms a partial mediating effect of message subject on the relationship between condition and argumentation (cf. Baron \& Kenny, 1986). Because this mediating effect of message subject might also exist for other dependent variables, they were checked for a possible correlation with message subject. Besides argumentation, message subject proved to correlate significantly with the percentage of confirmation $(r=-0.48, p=<0.01, n=36)$. Additional regression analyses revealed that this correlation is no longer significant when controlling for message subject (see Table 9), making the relationship between condition and the percentage of confirmations entirely mediated by message subject.

Table 7 Percentages of argumentations and confirmations by condition

\begin{tabular}{lll}
\hline & Forum discussion & Anchored discussion \\
\hline 3) Argumentation & 30 & 19 \\
4) Confirmation & 25 & 17 \\
\hline
\end{tabular}


Table 8 Regression analysis for condition and message subject predicting argumentation

\begin{tabular}{lllll}
\hline & $B$ & SE & $\beta$ & $p$ \\
\hline Step 1 condition & -1.09 & 0.03 & -0.49 & 0.003 \\
Step 2 condition message subject & -0.07 & 0.03 & -0.30 & 0.043 \\
& -0.21 & 0.06 & -0.49 & 0.001 \\
\hline
\end{tabular}

\section{Conclusion}

Turning back to the formulated research questions, several conclusions can be drawn. First, an increased percentage of meaning-oriented discussion, a more frequent referring to content, and a higher reported frequency of rereading relevant passages from the article indicate an affordance for anchored discussion to strengthen the link between discussion and study material. The cause for this enhanced link might be that the on-screen presence of the article, as well as the tool's specialized design, suggest to students that the discussion is to be focused on the meaning of the article. Put more generally, as conjectured in our description of anchored discussion, its design may have influenced students' perceptions of the collaborative goal and focused their collaborative intentions. Second, the system for anchored discussion seems to offer an increased communicative efficiency. With briefer referrals (demonstrative rather than comprehensive) and messages containing fewer self-clarifications than the system for regular forum discussion, participants need fewer words to express their ideas. On a more theoretical level, these brief referrals can be said to demonstrate the existence of a certain "frame of reference," as was expected. It is important to note, however, that this increase in communicative efficiency does not seem to lead to higher levels of mutual understanding, but rather seems to decrease the amount of effort that is required to reach this same level of mutual understanding. Third, discussion shows a different general character in the two systems, devoting relatively more attention to establishing social relationships and regulating the collaborative processes in the regular forum discussion and being more straightforward and "to-the-point" in the system for anchored discussion (also resulting in a greater number of messages). This absence of social and regulative coordination in the system for anchored discussion can be interpreted as a reduced need for establishing a "call back pressure," which might again have been caused by a greater task-directedness as influenced by the system's functional design. Fourth, regarding the constructive activities, we found some differences between the conditions. Both argumentations and confirmations are found relatively more often in the forum discussion. However, as this can be (partially) explained by a stronger orientation towards opinionoriented communication in the forum discussion, the relation between condition and the amount of argumentations and confirmations can be said to have been mediated by the subject of discussion.

Table 9 Regression analysis for condition and message subject predicting confirmation

\begin{tabular}{lllll}
\hline & $B$ & SE & $\beta$ & $p$ \\
\hline Step 1 condition & -0.073 & 0.03 & -0.35 & 0.036 \\
Step 2 condition message subject & -0.16 & 0.07 & -0.20 & 0.241 \\
& -0.04 & 0.03 & -0.41 & 0.017 \\
\hline
\end{tabular}




\section{Discussion}

Since we did not find any alternative reasons for the differences between the discussion in both systems, we contribute the results to the affordances of the two systems, and in particular to their functional design. Although the presence of meaning-oriented, efficient communication does not yet guarantee collaborative learning, the presented results do seem to confirm that the practical affordances of anchored discussion make it a good starting point for supporting (the early stages of) collaborative literature processing. On the other hand, to stimulate a more personal and critical discussion that might be more beneficial for participants with sufficient levels of understanding of the subject matter, traditional forum discussion might be better suited. Furthermore, while this study focused on answering four rather concrete, theory-driven, research questions, the presented results might also have some broader implications for the field of CSCL.

First, it seems possible to distinguish two different types of discussion. While the exchange of personal opinions has been shown to be related to a more argumentative kind of discussion, a strengthened link between discussion and study material seemed to be accompanied by a more constructive discussion, moving towards seeing the subject of discussion from a third-person perspective. Since being able to stand back from one's personal viewpoints and trying to get the meaning of a message or article might be a crucial element in the early stages of collaborative text comprehension, it seems useful to distinguish "argumentation for opinion" from "argumentation for interpretation"; if trying to stimulate the latter, a more soft approach might be needed (Veerman, 2000). This means it could be important to support students to take a vulnerable position and express even their "dumb" thoughts (Gay et al., 1999), as these can be seen as the only "building blocks" that are available to work with in trying to collaboratively advance their understanding of a text.

Second, an increased task-directedness in the system for anchored discussion was accompanied by a decreased amount of social and regulative communication. Taking a "grounding-is-functional" perspective on this finding would allow us to conclude that this condition presented students with sufficient levels of shared collaborative intentions and needed less explicit coordination of the process. Thus, although both socio-emotional and regulative processes are essential elements for successful CSCL Mäkitalo, Salo, Häkkinen \& Järvelä (2001), they do not necessarily need to manifest themselves in an explicit way. Especially in situations where sufficient levels of interdependency, trust and community have already been developed-for example in courses with abundant face-to-face contact between students-facilitating social and regulative communication might not be necessary and a more direct facilitation of the process of collaborative knowledge construction might be more productive. Furthermore, this emphasis on a direct facilitation of the collaborative task might also be a more effective way to increase students' motivation. As Järvenoja and Järvelä (2005) show, social processes play a less important role in determining student's motivation and emotion, than aspects that are related to the accomplishment of the task.

Finally, we suggest that for an increased availability of anchored discussion (university) practice, ${ }^{2}$ it could be useful to integrate functionality for anchored discussion into existing electronic learning environments.

\footnotetext{
${ }^{2}$ For inquiries about the practical availability of systems for anchored discussion, please contact j.vanderpol@ivlos.uu.nl, or find a working system at http://annotatie.ivlos.uu.nl/annotatie/ index.php.
} 


\section{Further research}

The obtained results encourage continuing research on and development of systems for anchored discussion. Furthermore, since the effects of anchored discussion on concrete learning results are still of a hypothesized nature, it would be useful to broaden and elaborate the techniques of analysis in order to assess the learning potential of certain patterns of interaction more closely. In particular, an operationalization of the relevancy and usefulness of replies would make it possible to evaluate the content of online discussion more accurately. Finally, gaining some insight into a possible way of facilitating certain concrete aspects of the collaborative processing of literature in asynchronous CMC by no means "solves" all of its limitations and still leaves many opportunities for further realizing the potential of online discussion for collaborative learning.

\section{References}

Baker, M., Hansen, T., Joiner, \& R., Traum, D. (1999). The role of grounding in collaborative learning tasks. In P. Dillenbourg (Ed.), Collaborative learning: Cognitive and computational approaches (pp. 31-63). Oxford: Pergamon.

Baron, R. M., \& Kenny, D. A. (1986). The moderator-mediator variable distinction in social psychological research: Conceptual, strategic, and statistical considerations. Journal of Personality and Social Psychology, 64, 708-722.

Bernheim Brush, A. J., Bargeron, D., Grudin, J., Borning, A., \& Gupta, A. (2002). Supporting interaction outside of class: Anchored discussion vs. discussion boards. In G. Stahl (Ed.), Computer support for collaborative learning: Foundations for a CSCL community. Proceedings of CSCL 2002 (pp. 425-434). Hillsdale, NY: Lawrence Erlbaum Associates.

Boekaerts, M., \& Simons, P. R. J. (1995). Leren en instructie: Psychologie van de leerling en het leerproces. (Learning and instruction: Psychology of the student and the learning process). Assen: Van Gorcum.

Cadiz, J. J., Gupta, A., \& Grudin, J. (2000). Using web annotations for asynchronous collaboration around documents. In D. G. Durand (Ed.), Proceedings of the ACM Conference on Computer Supported Cooperative Work 2000 (pp. 309-318). New York: ACM.

Clark, H. H., \& Brennan, S. E. (1991). Grounding in communication. In L. B. Resnick, J. M. Levine, \& S. D. Teasly (Eds.), Perspectives on socially shared cognition (pp. 127-149). Washington, DC: American Psychological Association.

Clark, D. B., Weinberger, A., Jucks, I., Spitulnik, M., \& Wallace, R. (2003). Designing effective science inquiry in text-based computer-supported collaborative learning environments. International Journal of Educational Policy, Research, \& Practice, 4(1), 55-82.

Davis, J. R., \& Huttenlocher, D. P. (1995). Shared annotation for cooperative learning. In J. L. Schnase \& E. L. Cunnius (Eds.), Proceedings of CSCL 1995 (pp. 84-88). Bloomington: Lawrence Erlbaum Associates.

De Laat, M. (2002). Network and content analysis in an online community discourse. In G. Stahl (Ed.), Computer support for collaborative learning: Foundations for a CSCL community. Proceedings of CSCL 2002 (pp. 625-626). Hillsdale, NY: Lawrence Erlbaum Associates.

Dillenbourg, P. (1999). What do you mean by collaborative learning? In P. Dillenbourg, (Ed.), Collaborative-learning: Cognitive and computational approaches (pp. 1-19). Oxford: Elsevier.

Fay N., Garrod, S. C., \& Carletta J. (2000). Group discussion as interactive dialogue or as serial monologue: The influence of group size. Psychological Science, 11(6), 481-486.

Fussell, S. R., \& Benimoff, N. I. (1995). Social and cognitive processes in interpersonal communication: Implications for advanced telecommunications technologies. Human Factors, $37,228-250$.

Gay, G., Sturgill, A., Martin, W., \& Huttenlocher, D (1999). Document-centered peer collabo- 
rations: An exploration of the educational uses of networked communication technologies. Journal of Computer-Mediated Communication, 4(3).

Gergle, D., Kraut, R. E., \& Fussell, S. R. (2004). Language efficiency and visual technology: Minimizing collaborative effort with visual information. Journal of Language and Social Psychology, 23, 491-517.

Gunawardena, C. N., Lowe, C. A., \& Anderson, T. (1997). Analysis of a global online debate and the development of an interaction analysis model for examining social construction of knowledge in computer conferencing. Journal of Educational Computing Research, 17(4), 397-431.

Guzdial, M., \& Turns, J. (2000). Effective discussion through a computer-mediated anchored forum. Journal of the Learning Sciences, 9(4), 437-469.

Herrmann, T., \& Kienle, A. (2003, January). Integration of communication, coordination and learning material $-A$ guide for the functionality of collaborative learning environments. Paper presented at the 36th Annual Hawaii International Conference on System Sciences, Hilton Waikoloa, Hawaii.

Hewitt, J., \& Teplovs, C. (1999). An analysis of growth patterns in computer conferencing threads. In C. Hoadley \& J. Roschelle (Eds.), Designing new media for a new millennium: Collaborative technology for learning, education, and training proceedings of CSCL 1999 (pp. 232-241). Mahwah, NJ: Lawrence Erlbaum Associates.

Hunt, R. A. (1998). Electronic discussions in learning and teaching: Why they don't work, and how they might. Connexions: The Newsletter of the International Society for the Exploration of Teaching Alternatives, 10(2), 1-7.

Järvelä, S. \& Häkkinen, P. (2000, June). Levels of Web-based discussion: Theory of perspectivetaking as a tool for analysing interaction. In B. Fishman \& S. O'Connor-Divelbiss (Eds.), Fourth International Conference on the Learning Sciences (pp. 22-26). Mahwah, NJ: Erlbaum.

Järvenoja, H., \& Järvelä, S. (2005). How students describe the sources of their emotional and motivational experiences during the learning process: A qualitative approach. Learning and Instruction, 15(5), 465-480.

Laurillard, D. (1993). Rethinking university teaching. London: Routeledge.

Lipponen, L. (2001). Computer-supported collaborative learning: From promises to reality. Unpublished doctoral dissertation, University of Turku, Turku, Finland.

Mäkitalo, K., Salo, P., Häkkinen, P., \& Järvelä, S. (2001, August). Analysing the mechanisms of a common ground in Web-based interaction. Paper presented at the JURE pre-conference of the 9th European Conference for Research on Learning and Instruction, Fribourg, Switzerland.

Marshall, C. C., \& Bernheim Brush, A. J. (2002, April). From personal to shared annotations. Paper presented at CHI 2002, Minneapolis, MN, USA.

McLoughlin, C., \& Luca, J. (2000). Cognitive engagement and higher order thinking through computer conferencing: We know why but do we know how? In A. Herrmann \& M. M. Kulski (Eds.), Flexible futures in tertiary teaching.Proceedings of the ninth annual teaching and learning forum. Perth, Australia: Curtin University of Technology.

Moon, J. A. (1999). Reflection in learning and professional development. London: Stylus.

Nokelainen, P., Miettinen, M., Kurhila, J., Floréen, P., \& Tirri, H. (2005). A shared document-based annotation tool to support learner-centered collaborative learning. British Journal of Educational Technology, 36(5), 757-770.

Pena-Shaff, J., \& Nicholls, C. (2004). Analyzing student interactions and meaning construction in computer bulletin board discussions. Computers \& Education, 42(3), 243-265.

Petraglia, J. (1998). Reality by design: The rhetoric and technology of authenticity in education. Mahwah, NY: Lawrence Erlbaum Associates.

Phillips, D. C. (1995). The good, the bad, and the ugly: The many faces of constructivism. Educational Researcher, 24(7), 5-12.

Pilkington, R. (2004). Developing discussion for learning. 2004 Journal of Computer Assisted Learning 20 (pp. 161-164). Oxford: Blackwell.

Schoonenboom, J. (2002). A template for discussing large texts on the web: The Pragglejaz site. British Journal of Educational Technology, 33(1), 103-107.

Schwartz, D. L., \& Lin, X. D. (2000). Computers, productive agency, and the effort after shared meaning. Journal of Computing in Higher Education, 12(2), 3-33.

Seltzer, W. (2000). Annotation engine (Technical report). Cambridge, MA: Harvard University, Berkman Center for Internet \& Society.

Simons, P. R. J., Van der Linden, J., \& Duffy, T. M. (2000). New learning: Three ways to learn in a new balance. In P. R. J. Simons, J. van der Linden, \& T. M. Duffy (Eds.), New learning (pp. 1-20). Dordrecht, the Netherlands: Kluwer. 
Sringam, C.,w \& Geer, R. (2000, September). An Investigation of an instrument for analysis of studentled electronic discussions. Paper presented at ASCILITE 2000, Coffs Harbour, Australia.

Stahl, G. (2000). A model of collaborative knowledge-building. In B. Fishman \& S. O'ConnorDivelbiss (Eds.), Proceedings of the fourth international conference of the learning sciences (pp. 70-77). Mahwah, NJ: Lawrence Erlbaum Associates.

Sumner, T., \& Buckingham Shum, S. (2001). JIME: An interactive journal for interactive media. First Monday, 6(2).

Suthers, D. (2005) Technology affordances for intersubjective learning: A thematic agenda for CSCL. In T. Koschmann, D. Suthers, \& T. W. Chan (Eds.), Computer supported collaborative learning 2005: The next 10 years (pp. 135-144). Mahwah, NJ: Lawrence Erlbaum Associates.

Takeda, T., \& Suthers, D. (2002, May). Online workspaces for annotation and discussion of documents. Poster session presented at WWW 2002, Honolulu, Hawaii.

Van der Pol, J. (2002, January). Identifying and modeling variables in complex CSCL-situations. Case study: The use of asynchronous electronic discussions. Paper presented the Workshop on Designing Computational Models of Collaborative Learning Interaction at CSCL2002, Boulder, CO.

Van der Pol, J., \& Admiraal, W. (2003). Het succesvol inzetten van asynchrone elektronsiche discussies. (Succesfully implementing asynchronous online discussions). Onderzoek van Onderwijs, 32(2), 26-31.

Van der Pol, J., Admiraal, W., \& Simons, P. R. J. (2006). Context enhancement for co-intentionality and co-reference. $A I$ \& society, 22(3).

Veerman, A. L. (2000). Computer-supported collaborative learning through argumentation. Unpublished doctoral dissertation, Department of Educational Science, Utrecht University, Utrecht, The Netherlands.

Veldhuis-Diermanse, E. (2002). CSCLearning? Unpublished doctoral dissertation, IVLOS, Utrecht University, Utrecht, The Netherlands.

Wan, D., \& Johnson, P. M. (1994). Experiences with Clare: A computer supported collaborative environment. International Journal of Human-Computer Systems, 41, 851-879.

Warschauer, M. (1997). Computer-mediated collaborative learning: Theory and practice. Modern Language Journal, 81(3), 470-481. 